\title{
Imagery and modality in paired-associate learning in the blind*
}

\author{
MICHAEL A. HANS $\dagger$ \\ State University of New York at Albany, Albany, New York 12203
}

\begin{abstract}
Congenitally blind Ss learned lists of 12 noun-noun word pairs in a paired-associates task, where the visual and auditory image-producing qualities of the stimulus and response terms of the pairs were varied. Performance on the paired-associates recall task was not affected by the imagery-producing quality of either the stimulus or response terms. However, the interaction of these qualities for the stimulus and response positions was significant, with 13 Ss recalling more pairs when the imagery-producing qualities of the stimulus and response terms matched. The data are interpreted as a rejection of a modality-specific imagery hypothesis.
\end{abstract}

Research concerning verbal learning of the blind has failed to give any real notion as to the qualities of words that give them meaning, thereby allowing the words to become part of the blind person's language. One approach is to consider Paivio's work on imagery. Schlaugel (1953) had noted that congenital and early blind persons did not possess imagery like sighted persons. Paivio (1971) hypothesized that words with only visual referents could not have any associated concreteness for the blind person, and would essentially be abstract. In order for words to possess concreteness, they would need to be associated with objects or events that could be sensed by other than visual means. In an unpublished study reported by Paivio (1971), Gardner and Paivio found that blind persons did not perform significantly better on a paired-associates task that used auditory image-producing words for the stimulus and response terms vs a situation utilizing visual image-producing words, or a combination of the two. In a later study, Paivio and Okovita (1971) presented Ss with two sets of noun pairs, one having words high in visual imagery and auditory imagery and the second set consisting of nouns high in.visual imagery but low in auditory imagery in a paired-associates task. The results supported their prediction that sighted Ss would do better on the high visual imagery, low auditory imagery word pairs than the blind Ss. Within the blind group, Ss performed better on the high visual imagery, high auditory imagery word pairs than on the high visual imagery, low auditory imagery word pairs; however, this difference was not significant for the sighted Ss. There was also no difference between the sighted and blind Ss' performance on the high visual imagery, high auditory imagery word pairs. Similar results were obtained using what were essentially pure visual vs pure auditory words,

\footnotetext{
* Special thanks are extended to Ronald Ley, State University of New York at Albany, for his criticisms of the original manuscript and subsequent revisions and for sponsoring the paper, and also to Joseph Kirstein, Albany Association of the Blind, and Charles VanDerKolk, State University of New York at Albany, for their cooperation in providing the Ss to make this study possible.

tRequests for reprints should be sent to the author at the Department of Educational Psychology, State University of New York at Albany, Albany, N.Y. 12203.
}

with the blind Ss recalling more auditory words than the sighted Ss and the reverse occurring for the visual words.

In another area of verbal learning, Paivio (1963) has explained differences in learning due to word position effects in terms of a conceptual-peg hypothesis. This hypothesis holds that the recall of a verbal unit may be initiated by an association with some other unit that preceded it in the learning trial through an image evoked to that stimulus word or unit. This predecessor, acting as a stimulus, evokes an image from which the recall of the response term is made. Lambert and Paivio (1956) found that word order was an important factor in serial learning of noun-adjective pairs. Paivio (1963) attempted to explain these earlier results by proposing that the imagery associated with the stimulus term was more important than the imagery associated with the response term. That is, differences in learning due to the abstractness or concreteness in words will be more prominent if the word serves as the stimulus term in the learning-recall situation. Paivio (1963) tested this conceptual-peg hypothesis in paired-associate learning by varying the concreteness of the stimulus terms in noun-adjective pairs. The results indicated that the concreteness of the stimulus term was an important factor in paired-associate learning when children were used as Ss, but the hypothesis was not confirmed for a sample of adults. Later, Paivio (1965) demonstrated that with noun-noun pairs, the concreteness of the stimulus term was more important than that of the response term. In an attempt to further isolate the effect due to each of the paired-associate learning terms, Yarmey and Paivio (1965) used noun-nonsense syllable pairs and reaffirmed the hypothesis that the concreteness of the stimulus term is more important than that of the response term.

The present study attempted to extend the study of the relationship of concreteness and verbal learning in blind persons to the conceptual-peg hypothesis. If the hypothesis obtains, then the concreteness of the stimulus term in paired-associate learning ought to be more important than that of the response term. Alternatively, it may be found that words that differ in 
Table 1

Mean Numbers of Words Recalled Over Four Trials for Each of the Four Visual-Auditory, Stimulus-Response.Combinations

\begin{tabular}{lcccc}
\hline \multirow{2}{*}{$\begin{array}{c}\text { Response } \\
\text { Term }\end{array}$} & \multicolumn{3}{c}{ Stimulus } & Term Imagery \\
Imagery & Mean & SD & & \multicolumn{2}{c}{$\begin{array}{c}\text { Auditory } \\
\text { Mean }\end{array}$} & SD \\
\hline Visual & 7.92 & 2.03 & 5.61 & 3.16 \\
Auditory & 6.84 & 2.67 & 8.30 & 3.38 \\
\hline
\end{tabular}

imagery have no effect on learning in the blind. Observations by J. Kirstein ${ }^{1}$ have suggested that many blind persons treat nearly all verbal material as abstract. Words that represent seemingly highly concrete objects (i.e., parts of the body, sidewalk obstacles) can be defined verbally, but often cannot be identified physically. These are called verbalisms.

More basically, then, the primary purpose of the present study was to retest the hypothesis that modality-specific imagery exists in the blind; the secondary purpose was to test the conceptual-peg hypothesis.

\section{METHOD}

\section{Design}

The hypotheses were tested by means of a 2 by 2 within-Ss experimental design, where the first factor was the imagery-producing quality of the stimulus term (i.e., visual or auditory) and the second factor was the imagery-producing quality of the response term (i.e., visual or auditory).

\section{Subjects}

The Ss were 13 congenitally blind clients and employees at the Albany Association of the Blind, Albany, N.Y. Most of the Ss were in various stages of rehabilitation in the Association's rehabilitation unit. Ages ranged from late teens to the $40 \mathrm{~s}$; there were 7 males and 6 females.

\section{Materials}

The materials consisted of 12 noun-noun pairs, in which the visual and auditory imagery-producing qualities of the stimulus and response term s were varied. All the words used were selected from the Paivio and Okovita (1971) study. The 12 pairs were divided into four groups. Three pairs (blood-tower, nun-maiden, and poster-meadow) had a high visual imagery-producing set of words in both the stimulus and response position; three pairs (thunder-squeak, song-applause, and echo-music) had high auditory imagery-producing words in both positions; and the remaining six pairs were further divided into two groups, one having high auditory imagery-producing words in the stimulus position and high visual imagery-producing words in the response position (click-sky, whisper-painting, and roar-star) and the other group having high auditory imagery-producing and high visual imagery-producing words in the opposite configuration (green, cough, hillside-tone, and rainbow-conversation). The order of presentation was random with respect to the type of noun-noun pairs.

\section{Procedure}

The Ss were given paired-associate learning instructions and then presented with the 12 noun-noun pairs on a tape recorder at a 4-sec rate. There were a total of four study-recall trial combinations. The criterion measure for each $\mathrm{S}$ was the number of units recalled for each particular combination of the imagery-producing qualities. The Ss were tested individually, and their responses were oral.

\section{RESULTS}

The mean number of response terms recalled over four trials for each of the four combinations of the imagery-producing qualities of the stimulus and response terms are given in Table 1. Neither of the main effects was significant; that is, the mean number of response terms recalled with respect to the stimulus position was 7.57 for high auditory imagery-producing stimulus terms and 6.76 for high visual imagery-producing stimulus terms $[F(1,12)=2.20, p>.05]$, and the mean number of response terms recalled with respect to the response term position was 6.96 for the high auditory imagery-producing response terms and 7.38 for the high visual imagery-producing response terms $[F(1,12)<1$, $p>.05]$. The Response Imagery by Stimulus Imagery interaction was significant $[\mathrm{F}(1,12)=12.44, \mathrm{p}<.01]$. Table 1 indicates that recall of high auditory imagery-producing response terms was greater when paired with a high auditory imagery-producing stimulus term than when paired with a high visual imagery-producing stimulus term.

\section{DISCUSSION}

The finding that the imagery-producing quality of both the stimulus and response terms had no effect on recall tends to reduce credence in a modality-specific imagery interpretation. The only possible source of support for modality-specific imagery occurs in the specific combination of high auditory imagery-producing words in both positions of a pair. The difference between the unimodal pair categories, which would have been predicted to be the largest difference by the modality-specific imagery hypothesis (and which provided a basis for Paivio and Okovita's support for their hypothesis), was the smallest of all differences between categories. Therefore, the data suggest that blind Ss do not utilize an auditory image to facilitate recall, but rather treat words with auditory referents much like words with visual referents. Confirmation of the conceptual-peg hypothesis was dependent upon the assumption that modality-specific imagery would be utilized; therefore, the failure for the hypothesis to be confirmed is not a basis for refuting it. The Stimulus Position by Response Position interaction, which had not been predicted by the modality-specific imagery hypothesis, may provide an alternate explanation. It may be that word pairs are interpreted as more meaningful if the two words have some relationship. Similar to Thorndike's (1931) concept of "belongedness," the blind Ss may have perceived significant relationships when the pairs had only one type of referent (i.e., auditory or visual). These unimodal words may have been classified together, resulting in a linkage between the paired nouns. Verbal reports of the Ss during the experimental trials often supported this idea. Some Ss indicated that several of these unimodal pairs were "very easy," while a few Ss commented that it was difficult for them to remember the responses for some of the bimodal pairs because "they didn't make any sense." These verbal reports tend to support the overall conclusion that blind Ss do not use modality-specific imagery in paired-associate learning. It may be illuminating to study the effects of associative reaction time, meaningfulness, and word familiarity on paired-associate learning in the blind. Paivio and Okovita's (1971) contention that these variables ought to be insignificant with blind $S s$ may be a bit presumptious. 


\section{REFERENCES}

Lambert, W. E., \& Paivio, A. The influence of noun-adjective order on learning. Canadian Journal of Psychology, 1956, 10 9-12.

Paivio, A. Learning of adjective-noun paired-associates as function of adjective-noun word order and noun abstractness Canadian Journal of Psychology, 1963, 17, 370-379.

Paivio, A. Abstractness, imagery, and meaningfulness in paired-associate learning. Journal of Verbal Learning \& Verbal Behavior, 1965, 4, 32-38.

Paivio, A. Imagery and verbal processes. New York: Holt, Rinehart \& Winston, 1971. P. 518.

Paivio, A., \& Okovita, H. W. Word imagery modalities and associative learning in blind and sighted subjects. Journal of Verbal Learning \& Verbal Behavior, 1971, 10, 506-510.

Thorndike, E. L. Human learning. New York: Appleton-Century-Crofts, 1931 .
Schlaugel, T. F. The dominant method of imagery in blind as compared to sighted adolescents. Journal of Genetic Psychology, 1953, 83, 265-277.

Yarmey, A. D., \& Paivio, A. Further evidence on the effects of word abstractness and meaningfulness in paired-associate learning. Psychonomic Science, 1965, 2, 307-308.

\section{NOTE}

1. Personal communication with J. Kirstein, Director, Rehabilitation Unit, Albany Association of the Blind, Albany, N.Y.

\title{
Wild caught vs inbred mouse strains: Differences in wheel-turn avoidance learning
}

DOMINIC J. ZERBOLIO, Jr.

\author{
University of Missouri, St. Louis, Missouri 63121
}

Three Bar Harbor inbred mouse strains; C57BL/6J, C3H/HeJ, and DBA/2J, and two wild caught but laboratory bred Peromyscus strains; $P$. leucopus and $P$. maniculatus bairdi, were compared over 8 days of acquisition training on a wheel-turn avoidance task. In the acquisition of the conditioned avoidance response, the Bar Harbor inbreds did not differ but were, as a group, significantly better than the wild caught strains. Differences in activity rate were also apparent, but do not account for the differences in avoidance performance. 